\title{
Salvinavia, eine Wortschöpfung des Naturforschers Chamisso
}

Von Carl Arbenz

Vor kurzem hat in dieser Zeitschrift (Gesnerus, Bd.41/1985, S. 325) Erwin H. Ackerknecht das bei Reimer, Berlin 1983, erschienene Buch angezeigt: Adelbert von Chamisso, «... und lassen gelten, was ich beobachtet habe». Naturwissenschaftliche Schriften mit Zeichnungen des Autors, herausgegeben von Ruth Schneebeli-Graf. Übersetzung und Bearbeitung der lateinischen Texte von Friedrich Markgraf.

Die Autoren dieses Buches ersuchten mich noch vor dessen Drucklegung um die Gefälligkeit zu überprüfen, ob ein Wort Salvinavia, das sie in einem lateinischen Text Chamissos vorgefunden, in der römischen Antike bezeugt sei. Auf meine Rückfrage nach Vorkommen und Zusammenhang lieferte mir der inzwischen verstorbene Prof. Markgraf den vollständigen Wortlaut der Stelle:

Die XXX.Julii e portu Petropolitano Corona profectum est. Ipse navem conscendi Hafniae die IX. Augusti. Favente Salvinavia tres per integros annos peregrinati sumus, vix per tertiam huius temporis partem, ancoris fixis, exterae nobis patuere Florae sedes.

«Am 30. Juli stach das Schiff aus dem Petersburger Hafen Kronstadt in See. Ich selbst bestieg es am 9. August zu Kopenhagen. Daraufhin sind wir unter der Obhut der Schutzgöttin der Schiffe volle drei Jahre lang umhergesegelt, doch konnten wir kaum während eines Dritteils der Zeit, wenn wir einmal vor Anker lagen, die fremdländischen Pflanzenstandorte aufsuchen.» ${ }^{1}$

Diese drei Sätze stehen in Chamissos kurzer Vorrede zum pflanzenkundlichen Rechenschaftsbericht, den er in Gemeinschaft mit dem Botaniker Dietrich Franz von Schlechtendal über den naturwissenschaftlichen Ertrag der Romanzowschen Forschungsexpedition in der neuen, von ihnen begründeten Zeitschrift «Linnaea», Bd.I, Berlin 1826, veröffentlicht hat. Es handelt sich bei der Expedition um das von Zar Alexander I. begünstigte und vom ehemaligen russischen Reichskanzler Graf Romanzow ausgerüstete Privatunternehmen der Jahre 1815-18, das die Erkundung der Beringstraße und ihrer Umwelt zum Hauptzweck hatte und an dem Chamisso auf Initiative seines Berliner Freundes und späteren Erstbiographen Julius Eduard Hitzig hatte teilnehmen können. 
Das Schiff war beim Stapellauf im finnischen Seehafen Åbo auf den Namen «Rurik» getauft worden, des legendenumwobenen Königs des Reiches von Nowgorod aus dem 10. Jahrhundert, und unterstand alsdann dem Kommando des Kapitänleutnants Otto von Kotzebue. In der dreijährigen Seereise war die Umfahrung beider Südkaps notwendig miteingeschlossen, so daß den Forschern nicht nur ein nordisches, sondern ein weltweites Gebiet zur Verfügung stand.

Nach der Rückkehr erhielt Chamisso, in Anerkennung seiner ausgewiesenen Forschungsarbeit Ehrendoktor und Mitglied mehrerer Akademien geworden, in Berlin eine Stelle als Kustos des Botanischen Gartens zu Schöneberg. Mit dem Rückhalt dieses Amtes und der fachmännischen Mitwirkung Schlechtendals vermochte er die erwartete Auswertung der biologischen Erkenntnisse der Entdeckungsreise im Verlauf einiger Jahre aufzuarbeiten, und zu deren systematischer Darlegung schufen sich die beiden Gelehrten ihr eigenes Organ mit eben der genannten Zeitschrift «Linnaea», deren lateinisch geschriebene Aufsätze sich durch einen wohlgeschulten Stil auszeichnen, den sich ihre Verfasser nur in ausgiebigster Quellenlektüre erworben haben können ${ }^{2}$.

So verrät auch der in Rede stehende zweigliedrige Ausdruck «favente Salvinavia» als Ganzes eine frappierende Vertrautheit mit klassischen lateinischen Wendungen samt ihren Belegstellen, in unserm Falle z.B. favente dea (scil. Hecate, Venere, Victoria) oder auch im Plural faventibus dis, dazu in eng verwandter Situation die Stelle bei Ovid, Metamorphosen XV, 49: ... ventisque faventibus aequor / navigat Ionium Lacedaemoniumque Tarentum / praeterit et Sybarim etc. «... und so befährt er (der in die Fremde Entsandte) mit der Winde Gunst das Jonische Meer» etc.

Hatte sich somit die Formel nach Inhalt und Gestalt in der lateinischen Sprache als wohlverankert erwiesen, so lag es nahe, ein Gleiches auch für den darin enthaltenen Namen Salvinavia anzunehmen. Dementsprechend schreibt August C.Mahr, Professor für Germanistik an der Ohio State University in Columbus ${ }^{3}$, in seinem Vermerk zur Stelle: p. 127, Anm. (2 a) "a Roman goddess, protectress of ships at sea". Eine Auskunft über die Quelle seiner Aussage bei ihm einzuholen fiel dahin, da er nach Angabe der Universität vor über zwanzig Jahren gestorben ist. Aber Mahr hat den Sachverhalt offenbar nicht weiter überprüft. Denn laut Egidio Forcellini (1688-1768), Totius Latinitatis Lexicon, Padua ${ }^{1} 1771,{ }^{2} 1805$, ist die Vokabel schlechterdings nicht belegt, ein Befund, der sich in den Materialien des Thesaurus Linguae Latinae in München bestätigt ${ }^{4}$. So steht denn in den 
Anmerkungen auf Seite 34 des Buches, unter Berufung auf das Ergebnis meiner Kontrolle, eine Salvinavia sei - entgegen der Anmerkung Mahrs - als römische Gottheit nicht bezeugt, und der Ursprung des Wortes blieb somit im dunkeln.

Ein halbes Jahr später - das Buch war bereits im Druck - stieß ich bei der Beschäftigung mit lateinischen Inschriftformeln auf die drei gleichartigen Weihinschriften im Corpus Inscriptionum Latinarum CIL VI Nr. 492, 493 und $494^{5}$, die alle das Dativobjekt Navisalviae aufweisen als Bezeichnung einer Heilsgottheit der Hochseeschiffahrt, der eine begünstigte Person einen Altar für Gewährung von Schutz oder Nothilfe gespendet hat.

Dieser seltsame Name, bis ins 18. Jahrhundert ein unbekanntes Wort, hat Chamisso offensichtlich das Muster geboten zu seinem Gottheitsnamen Salvinavia, den er für seine thematisch verwandte Stelle neu geschaffen hat, indem er eine Art Schüttelreim bildete.

Wie aber ist Chamisso zur Kenntnis dieses Wortes gelangt, mit dem er sich den Scherz geleistet? Zur Beantwortung dieser unausweichlichen Frage müssen wir uns zunächst eingehender mit den drei Inschriften befassen, die allesamt das Stichwort zu Chamissos erfinderischem Wortspiel geliefert haben. Zuerst die Inschrift Nr. 494. Sie könnte hier zwar füglich übergangen werden, weil sie, wie sich sofort herausstellt, im Gegensatz zu den beiden anderen Inschriften in keinem für Chamisso attraktiven Zusammenhang steht. Indessen wäre es doch eine unangebrachte Unterlassung, bei dieser Gelegenheit die bisherigen Inschriftenkommentare nicht mit der Mitteilung des Deutschen Archäologischen Instituts in Rom zu ergänzen, daß der Marmoraltar, auf dem sie stand, mit den andern Beständen der 1822 erfolgten Aufhebung der Sammlung Borgia in Velletri möglicherweise nach Neapel in das Museo Reale gelangt sei. Auf meine sofortige Erkundigung daselbst ist aber bisher noch kein Bescheid eingetroffen.

Die beiden anderen Altäre mit den Inschriften Nr.492 und 493 sind laut Ficoroni ${ }^{6}$ gleichzeitig in altem Uferschwemmsand des Tibers im Weinberg Sforza-Cesarini zur Zeit des Papstes Clemens XI. (1700-21) gefunden worden, an einer La Marmorata heißenden Örtlichkeit zu Füßen des Aventin, wo sich im Altertum Schiffsanlegestellen, Lagerhallen und Heiligtümer für Dankesbezeugungen nach glücklicher Heimkehr befanden. Der Inschriftstein Nr.493 muß laut Guasco, seinerzeit Kurator des Kapitolinischen Museums ${ }^{7}$, spätestens im Jahre 1729 oder bald darauf nach Verona überführt worden sein, als Geschenk seines Finders Ficoroni an den ihm befreundeten Francesco Bianchini von Verona (1662-1729), der im hohen 
Range eines Prelato domestico (praesul) unter Clemens XI. zumeist in Rom tätig war. Das seit 1740 (siehe Anmerkung 13) aufgeführte Inventarstück des Museo Veronese findet sich heute als Votivtafel an einer Wand des neu eingerichteten Museo Lapidario ${ }^{8}$.

Der Altar mit der Inschrift Nr. 492 dagegen wurde zuerst in der Vatikanischen Bibliothek aufbewahrt, dann aber $1740^{9}$ an das Kapitolinische Museum abgegeben, wo er sich noch heute an dem von den Katalogen angezeigten Standort befindet.

Von der Veroneser Inschrift Nr. 493 läßt sich hier absehen; sie trägt, wenn auch in anderer Anordnung, dieselben Namen wie die Inschrift Nr.492, die wir hier wiedergeben unter Hinweis auf die Abbildung ${ }^{10}$ :

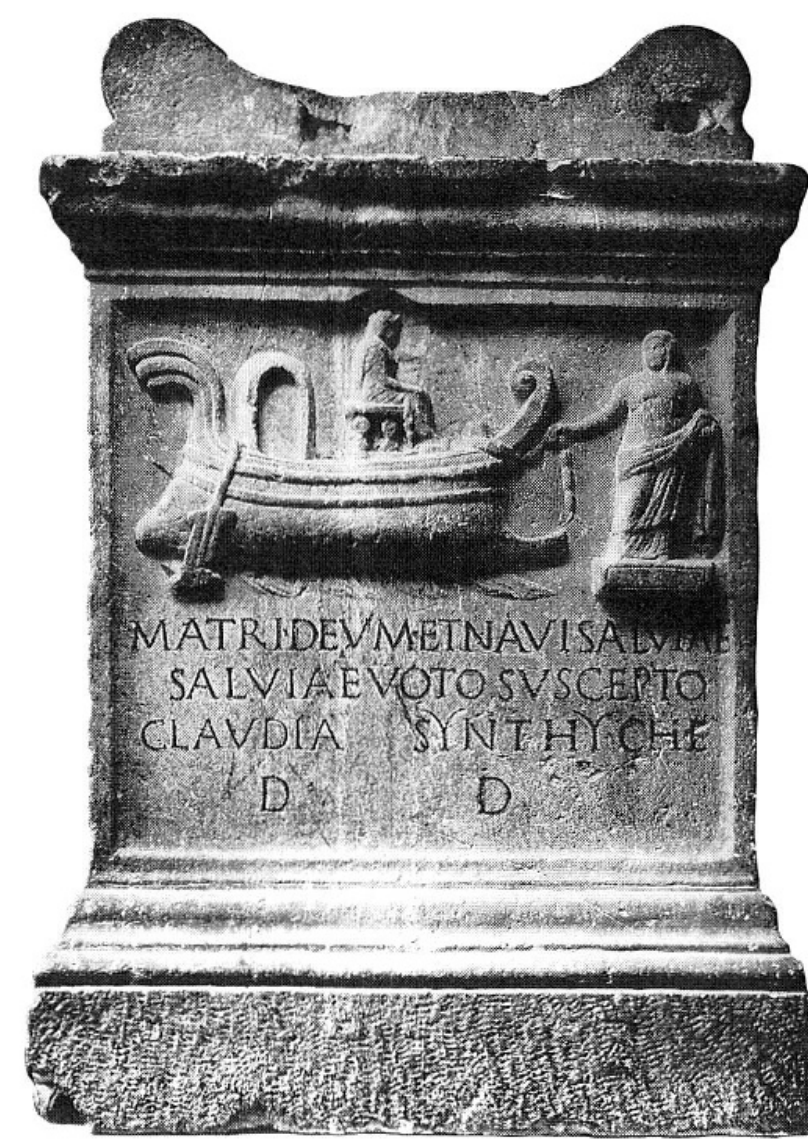

MATRI DEUM ET NAVISALVIAE

SALVIAE VOTO SUSCEPTO

CLAUDIA SYNTHYCHE

D D

$$
\begin{gathered}
\text { «(Diesen Altar hat) } \\
\text { der Mutter der Götter } \\
\text { und } \\
\text { der Behüterin der Schiffe } \\
\text { nach getanem Gelübde } \\
\text { Claudia Syntyche } \\
\text { zu Geschenk gegeben» } \\
\text { (D D = DONO DEDIT) }
\end{gathered}
$$

Der Altar wird aus Gründen seines Stils etwa der Zeit des Kaisers Claudius (41-54 n. Chr.) zugewiesen. Name und Formel am Schluß gestatten die Annahme, daß eine griechische Haussklavin namens Syntyche aus einer claudischen Familie zum Dank für ihre Freilassung gemäß feierlich gelobter 
Verpflichtung den beiden Gottheiten oder wahrscheinlicher ein und derselben Gottheit Cybele mit Bezug auf ihre doppelte Funktion als Göttermutter und Schiffsbehüterin diesen Altar gestiftet habe.

Die Wiederholung SALVIAE in der 2.Zeile der Inschrift dürfte ihren Grund entgegen geäußerten Zweifeln doch wohl in mangelnder Aufmerksamkeit des Steinmetzen haben, der ja auch mit der Rechtschreibung des griechischen Namens Syntyche seine kleine Mühe bekundet hat. Zudem war es vermutlich derselbe Mann, dem auch die beiden Unachtsamkeiten in der gleichgearteten Inschrift Nr.493 unterlaufen sind: DEU statt DEUM, SINTICHE statt SYNTYCHE.

Welche Bewandtnis es mit dem zuvor nie gehörten Namen Navisalvia hat, darüber gibt das Relief über der Inschrift Aufschluß: Das Bild stellt das heilige Schiff dar, mit dem im Jahre $204 \mathrm{v}$. Chr. auf eingeholte Weisung der cumäischen Sibylle die Göttermutter aus Kleinasien nach Rom überführt worden ist, um unter der Schutzmacht ihrer Gegenwart den Endsieg über den Karthager Hannibal sicherzustellen. Zu ihrem feierlichen Empfang in Ostia wurden «als bester Mann und beste Frau» der vornehme Scipio Nasica und die Vestalin Claudia Quinta bestimmt. Als das Schiff im Sand der Tibermündung steckenblieb, soll Claudia, deren Legitimation für den hohen Auftrag unter dem Verdacht unreinen Lebenswandels angezweifelt wurde, beschwörend ausgerufen haben: «So wahr ich meine Reinheit bewahrt habe, mag mir die Göttermutter helfen, das Schiff alleine wieder flottzubringen!» 11

Das Bild zeigt Claudia, wie sie das Schiff mit ihrer priesterlichen Leibkordel (infula) am Bug ziehend in Bewegung setzt. Cybele thront verschleiert und tympanon-bewehrt auf einem Podest des Fahrzeugs. Die Schmalseiten des Altars zeigen eine phrygische Mütze bzw. einen Hirtenstab.

Seither offenbar wird Cybele sowohl im Hinblick auf ihre Rolle als Siegverleiherin wie auch mit Bezug auf dieses Wundergeschehen von den Claudiern als Hüterin des Adelsgeschlechts verehrt, und in diesem Sinne ist wohl die Widmung zu verstehen.

Als geschlossenes Wort ${ }^{12}$ gehört Navisalvia der Titel-, Berufs- und Eigennamensphäre an und läuft nach dem Muster von mulo-medicus, domicurius, viti-sator etc. («Ross-Arzt», «Haus-Walter», «Reben-Pflanzer»), also: Dea, quae navem oder naves salvat $=$ conservat («die Göttin, die das Schiff heil ans Ziel bringt», die «Schiffs-Behüterin»). Es sei hier noch der Hinweis gestattet, daß die heidnische Göttermutter Cybele auch in ihrer 
Rolle als «Schiffsbehüterin» später durch die christliche Mutter Gottes abgelöst worden ist, unter deren Schutz z. B. Columbus seine Erkundung des westlichen Seewegs gestellt hatte.

Die bisher unbekannte Vokabel Navisalvia fand nun samt ihrem Kontexte vorerst Eingang in die neuen Inschriften-Publikationen des 18. Jahrhunderts. Das umfassendste Werk war damals die Sammlung des berühmten Italieners Lodovico Antonio Muratori (Modena 1672-1750), der die große Masse neuentdeckten Materials unter dem Titel «Novus Thesaurus veterum inscriptionum» in 4 Folianten veröffentlichte (Mailand 1739-42), sich aber dabei auf bereits vorhandene, örtlich begrenzte oder gar unedierte Privatkollektionen stützte. Bei Muratori steht die Inschrift von Velletri in Bd. I, pag. XCVIII, Nr. 3, unter Berufung auf eine Zuschrift des Benediktinerpriors Ginanni zu Ravenna, die beiden Inschriften von Rom und Verona dagegen erst in der Appendix, Bd.IV, pag. MCMLXXXV, Nr. 5 und 6, unter Berufung auf eine lokalhistorische Schrift von Marchese Scipione di Maffei ${ }^{13}$.

Die Aufnahme des Wortes in die Lexikographie ließ verständlicherweise noch eine Weile auf sich warten. Zuerst erschien es in der 7.Ausgabe des Großwerks des Humanisten Calepinus (Ambrogio Calepino), VII Linguarum Lexicon Latinum, Bd.II, Padua 1752, im deutschsprachigen Raume erstmals volle 36 Jahre später, im damals maßgebend gewordenen Werk: Immanuel Johann Gerhard Schellers «möglichst vollständiges» lateinischdeutsches Wörterbuch in 3 Bänden, das laut Hoffmann ${ }^{14}$ auch in der Lünemannschen Bearbeitung ab 1804 «sich die allgemeinste Geltung» erworben habe. Das Schellersche Lexikon, 1783 erstmals erschienen, führt die Vokabel Navisalvia seit der 2. Auflage an (Leipzig 1788) und beruft sich wie schon der erwähnte Calepinus allein auf Muratoris «Thesaurus». Als somit unser Wortmuster Navisalvia Eingang in die deutsche Lexikographie gefunden hatte, war Chamisso bereits am Leben.

Aber auch die drei Navisalvia-Inschriften mit ihrem gesamten Wortlaut wurden außerhalb Italiens bekannt gemacht und besprochen ${ }^{15}$, und die Nachricht vom Fund der Altäre wird wegen des Bezugs ihrer Reliefbilder auf das spektakuläre historische Ereignis wie ein Lauffeuer durch die Gelehrtenwelt der Zeit gegangen sein, so daß das Wissen darum zu einem vielerörterten Bestandteil damaliger Unterrichtung geworden sein dürfte, was Chamisso kaum entgangen ist. Man denke nur etwa an die publizistische Tätigkeit Winckelmanns während seines langen römischen Aufenthalts seit 1755, an die archäologischen Interessen mehrerer Päpste des 18. Jahrhunderts und an 
den Widerhall, den die immer neuen Fundmeldungen bei der europäischen Intelligenz erweckt haben.

So wird auch Chamisso in seinem Berliner Leben von den drei Inschriften mit ihrem markanten Zusammenhang Kenntnis bekommen haben. Das ist durchaus nicht unwahrscheinlich, und damit würde es denn auch plausibel, daß Chamisso den auffälligen, Gesprächsstoff gewordenen Namen gespeichert und bei adäquater Gelegenheit in Gestalt eines Schüttelreimes überraschend wieder zum Vorschein gebracht hat.

Zur Stütze einer solchen These muß hier auf seine umfassende Bildung und Ubiquität verwiesen werden, die er sich von Stufe zu Stufe erworben hatte. Zuerst einmal hatte der Emigrantensohn aus altfranzösischem Adel am hugenottisch beherrschten Collège français in Berlin bei hervorragenden Lehrkräften eine zwar kurze, aber dauerhafte Grundschulung genossen. Dann, mit zwanzig Jahren im Leutnantsgrad Kommandant der Brandenburger Torwache geworden, benützte er seine reichliche Freizeit zu einer intensiven Fortbildung in Griechisch und Latein und betrieb daneben ein ernsthaftes Selbststudium auf naturwissenschaftlichem Gebiet, auf dem er sich später an der 1810 eröffneten Berliner Universität als Student der Medizin weiterbildete. Er war somit bei weitem nicht nur der Verfasser des «Schlemihl» und der Poet, dessen beste und beliebteste Gedichte lange unsere Schulbücher belebten; er war vorwiegend Naturforscher und als solcher ein kenntnisreicher Pflanzensystematiker mit wachem Spürsinn und prinzipiellem Denkvermögen. Aber er war gleichzeitig von erstaunlicher Belesenheit in allem Wesentlichen der altklassischen Literatur und schrieb ein recht gewandtes Latein, wie so viele Vertreter der damaligen höheren Bildungsschicht. In seinem frühromantischen Verkehrskreise, der ihm Begegnungen mit den Brüdern Humboldt und Schlegel, Tieck, Fichte und Schleiermacher erschloß, war alles zu vernehmen, was sich an Nennenswertem im Geistesleben zutrug, und was Chamissos vorauszusetzende Bekanntschaft mit dem Fund der drei behandelten Inschriften betrifft, so muß hier daran erinnert werden, daß Chamisso im Winter 1803/04 bei A. W. Schlegel die «Vorlesungen über schöne Literatur und Kunst» hörte, deren erster Zyklus sich mit der antiken Literatur befaßte und die Geschichte von der Versetzung der Göttermutter mitenthalten haben mochte.

Als Romantiker war Chamisso aber auch sprachwissenschaftlich bewandert, wie sich deutlich erweist durch die Art, wie er die Erforschung der Südseesprachen während der «Rurik»-Expedition betrieb. Er legte Wortlisten an, schloß Wortstrukturen auf und entwarf Grammatiken. 
Sein Latein war ihm allgegenwärtig. So macht er auch im «Schlemihl», genau wie Eichendorff im «Taugenichts», gegebenenorts gern vom Latein Gebrauch, zumal in gespielt gravitätischen Szenarien und oft in burschikospathetischem Unernst. Als illustrative Beispiele geben wir hier einige Stellen aus dem 11. Abschnitt zum besten:

Daselbst nennt er den Ort, «worin er krank lag» und zu neuem Leben genas, schalkhaft das (Hospitium) Schlemihlium. Dann läßt er Schlemihl gleich einer Vision seiner eigenen künftigen Abhandlungen - das Ergebnis der erträumten Siebenmeilenstiefelreise, auf der nebst der Beringstraße das hunderttorige Theben und die Herkulessäulen angelaufen werden, vorwegnehmen mit dem Satze: «Meine Historia stirpium plantarum utriusque orbis steht da als ein großes Fragment der Flora universalis terrae und als ein Glied meines Systema naturae.» Und Chamissos Erzählung endet mit dem Schlußwort der lateinischen Codices: Explicit.

Des weitern können an verwendeten Formeln aus seinem deutsch geschriebenen Reisebericht von 1836 angeführt werden: votum solvens (30.10.1815), Relata refero (26.10.1816), horribile dictu! (10.1.1817) und Mitte August 1817 eine Bemerkung ad vocem Kitung, i.e. pediculus, ein alëutisches Wort für Kleinlaus. Einen ganzen Horazvers (Epist. I, 17, 36) zitiert er zu seinem Aufenthalt in Manila im Januar 1818: «Die chinesische Vorstadt ist für den anziehend, der das Reich der Mitte nicht betreten hat. Non cuivis homini contingit adire Corinthum.»

Chamissos sprachspielerische Neigungen werden an einigen wenigen Stellen seiner Prosa aufs deutlichste greifbar: Erstens einmal in der Wahl des Namens Schlemihl, der soviel bedeutet wie «ewiger Pechvogel» und aus dem Jiddischen hergeholt ist, sodann an einigen Sätzen der ersten zwei Abschnitte, nämlich

mit dem Zeugma: «Er (Herr Thomas John) brach das Siegel auf und das Gespräch nicht ab, das sich auf den Reichtum lenkte.»

mit der Kehrform: «Ich fürchtete mich fast noch mehr vor den Herren Bedienten als vor den bedienten Herren.»

oder mit Chiasmen in der Weise des Beispiels: «Man sprach zuweilen von leichtsinnigen Dingen wichtig, von wichtigen öfters leichtsinnig.» Und schließlich bei der Prägung spaßiger Analogieformen wie «der Heranwüchsling» nach dem Muster von Pflegling, Jüngling u. dgl.

Habe ich bei dem Versuch, die Richtigkeit unserer Vermutung zu erweisen, vorläufig nur die Allgegenwart des Lateins in Chamissos Schrifttum aufgezeigt und überdies die kundige und humorige Art seines Umgangs 
mit der Sprachform sichtbar werden lassen, so scheint mir darüber hinaus seine Vertrautheit mit den antiken Stoffen, für unsere Frage speziell mit den Inhalten der Hauptwerke Ovids, von ausschlaggebendem Gewicht zu sein.

Abgesehen von vereinzelten Anspielungen an Inhalte der Metamorphosen wie z. B. die Geschichte der Arethusa (V, 572-641) ist es vor allem seine Selbstbiographie, die ihn als Leser Ovids erkennen läßt. Diese Selbstbiographie steht in dem obenerwähnten Reisebericht von 1836, und zwar «Einleitend» gleich nach seinem Vorwort. Chamisso beginnt, wie Ovid in den Tristien IV, 10, $1 \mathrm{f}$. nach bekanntem Wortlaut: «Ille ego, qui fuerim, ..., accipe posteritas!» mit dem Satze, der Leser solle «zuvörderst erfahren, wer ich bin», und fährt alsdann in gleichartigem Aufbau fort mit der Aufreihung der markantesten Lebensdaten, alles im Hinblick auf seine trübe Zukunft als «vaterlandsloser Gesell», als der er sich in jenen Jahren der napoleonischen Peripetie vorgekommen, so daß er sich mächtig erinnert fühlte an seinen ebenso vaterlandslos gewordenen, in die Ferne des Schwarzen Meeres verbannten Schicksalsgenossen Ovid.

Chamisso muß auch Ovids Fasten gekannt haben und damit die Erzählung über die berühmte Seefahrt der Cybele vom Jahre 204 v. Chr. im Buch IV, 247-348. Im Rückblick auf seine eigene Weltumsegelung stiegen ihm wieder die Bilder auf von jenem ebenso hochgemuten nationalen Unternehmen der Antike, das, genauso wie die stationenreiche Erkundungsfahrt des «Rurik», das sieghafte Ende eines langwierigen, epochemachenden Krieges markierte.

Und die beiden Expeditionen hatten hinwiederum ihre analogen Akzente: Der eigens zu dem hohen Zwecke veranlaßte Schiffsbau mit dem ausgesuchten Holze, hier am Ida-Gebirge, dort im schwedenfinnischen Hafen Åbo; die Bergung beider Schiffe je im hauptstädtischen Binnengewässer des Tibers bzw. der Newa; und für die verbindende Mitte das Wirken der behütenden Hand der den beiden Besatzungen gewogenen Gottheiten: Cybele Navisalvia und - eben - Salvinavia.

Die dem von Chamisso gestalteten Wort Salvinavia formal und gedanklich zunächst stehende Bezeichnung (Venus dea) Verticordia (= quae cor oder corda vertit) steht übrigens in verbal abgewandelter Form kurz vor dem Cybele-Abschnitt a.a.O. Vers 160: verso corde; der Zuname selbst bei Valerius Maximus VIII, 15, 12:

Merito virorum commemorationi Sulpicia.... adicitur, quae, cum senatus censuisset, ut Veneris Verticordiae simulacrum consecraretur, ..., cunctis castitate praelata est. 
«Verdientermaßen pflegt man der Erwähnung großer Männer auch den Namen der Sulpicia beizufügen, die, als der Senat beantragt hatte, es solle ein Götterbild der Venus als (Sinneswenderin) geweiht werden, allen andern bezüglich Enthaltsamkeit vorgezogen worden ist.»

Wir haben also in einem Abstand von weniger als hundert Versen bei Ovid zwei analoge Szenen vor uns, die getragen sind, die eine vom Wirken der Venus Verticordia, die andere von dem der Cybele, der auf unsern drei Inschriften unter dem Namen Navisalvia gehuldigt wird.

Zweitausend Jahre später, nach Wiederentdeckung der inzwischen verschollenen Bezeichnung Navisalvia, rückt das zugehörige Großereignis plötzlich wieder ins Blickfeld der klassisch gebildeten Welt, so daß es einen Chamisso reizt, das Seeunternehmen des Zarenreichs, an dem er teilgenommen, damit in Vergleich zu bringen. Ovids dichterische Fastenchronik über die alte Mär vor Augen, unterstellt er, wie eingangs erwähnt, das eigene Großerlebnis in seinem Bericht dem Schutz einer entsprechenden Gottheit und schafft dafür die abgewandelte Gestalt einer Salvinavia, indem er die Cybele Navisalvia und die (Venus) Verticordia in seiner Wortbildung witzig kombiniert.

\section{Anmerkungen}

1 Die Übersetzung weicht zufolge unterschiedlicher Auffassung des Adjektivs exterus von derjenigen Markgrafs ein wenig ab, welche lautet: «Kaum ein Drittel dieser Zeit, während wir vor Anker lagen, standen uns die außerhalb liegenden Stätten der Flora offen.»

2 Die Zeitschrift bestand über Chamissos und Schlechtendals Tod (1838 bzw. 1866) mit insgesamt 43 Bänden bis 1882 fort. Siehe die umfassende Bibliographie von Günther Schmid, Chamisso als Naturforscher, Leipzig 1942. Diesbezügliche Angabe auf S. 60.

3 Es handelt sich um die stattliche Huldingungsschrift an seine Lieblingsstadt San Francisco unter dem Titel "The Visit of the 'Rurik' to San Francisco in 1816", herausgegeben im Rahmen der Stanford University Publications, Oxford 1932, in welcher er sämtliche Kalifornien betreffenden Teile aller Expeditionsberichte zweisprachig zusammengestellt hat, wozu selbstverständlich auch die eingangs erwähnte «Vorrede» Chamissos zum ersten lateinischen Fachbericht von 1826 gehört. Siehe Günther Schmid, a. a. O., Nr. 378, S. 158.

4 Die Auskunft verdanke ich der Vermittlung von Dr. phil.et iur. Alfons Bürge, München. Weder der Band mit $\mathrm{N}$ - noch derjenige mit $\mathrm{S}$ - ist bisher erschienen.

5 In der verbreiteten Sammlung von Hermann Dessau, Inscriptiones Latinae selectae II, 1, Nr. 4096-98. Berlin ${ }^{3} 1962$.

6 Francesco Ficoroni (1664-1747), Vestigia di Roma Antica, p. 149. 
7 Franciscus Eugenius Guasco, Musei Capitolini antiquae inscriptiones, nunc primum coniunctim editae, I, p. 35 (Nr.15), Rom 1775: Lapis iste, quem doctissimo Praesuli Francisco Blanchinio tradidit ipse Ficoronius, extat adhuc in Museo Veronensi.

8 Ich verdanke die Nachprüfung und die Beschaffung von Photographien einem Museumsbesuch von Dr. Ursula Oberli, Bülach, im Oktober 1986.

9 Laut Helbig/Speier, Führer durch die öffentlichen Sammlungen klassischer Altertümer in Rom, 2. Bd: die städtischen Sammlungen.

Kapitolinisches Museum, Katalognummer 1175, S.24; Standort: Stanza Terrena a Sinistra I, links der Eingangstür, bestätigt durch Dr. R. Neudecker, Assistent am Deutschen Archäologischen Institut in Rom, mit Brief vom 2. 10.1986. Vermerk: oft geschlossen und daher nicht immer zugänglich.

10 Bewilligte Reproduktion des Abzugs eines Negativs aus den Beständen des genannten Instituts.

11 Das gewichtige Ereignis wird in der lateinischen Literatur bis in die späte Kaiserzeit etwa zwanzigmal erwähnt und behandelt.

12 Für getrennte Schreibweise Navi Salviae, zum Nominativ Navis Salvia nach Mustern wie Urbs Salvia «Heilstadt» (vgl. Heilbronn), für welche namhafte Verfechter eingetreten sind, liefern die Inschriften im Original keinerlei graphischen Anhaltspunkte.

13 Osservazioni letterarie, t. VI, p. 292, Verona 1740, eine Stelle, auf die sich Maffei selber beruft in seinem Katalog von 1749: Museum Veronense, p.XC, Nr. 1, während er die Votivtafel in seinem großen Werk «Verona illustrata» in 4. Teilen, erschienen 1731/32, noch nicht erwähnt. Dieses reichdotierte Werk ist als kulturgeschichtliche Fundgrube zweimal je fünfbändig wieder herausgegeben worden, erstmals 1825-27 in Mailand und in unverändertem Nachdruck 1977 in Rom.

14 S. F. W. Hoffmann, Handbuch zur Bücherkunde für Lehre und Studium der beiden alten klassischen und deutschen Sprache, s.n. S. 22. Leipzig 1838.

15 Im CIL wird z. B. auf den Engländer Breval verwiesen, der von dem Fund mit der Bemerkung Notiz nahm: Discovered not many years since.

Nebst den in den Anmerkungen 4, 8 und 9 bereits genannten Personen danke ich den Herren Dr. B.Glaus, Leiter der wissensehaftshistorischen Sammlungen der ETH Zürich, und lic. phil. P. Simek, Oberbibliothekar des Botanischen Instituts der Universität Zürich, für die ErschlieBung bedingt erhältlicher Quellentexte; Prof.Dr. P. Wegelin, Direktor der St.Gallischen Kantonsbibliothek Vadiana, und Dr. F. Hieronymus, Fachreferent an der Universitätsbibliothek Basel, für ihre bereitwillige Mithilfe zur Eruierung ältester Lateinwörterbücher; H. Amberg, Stadtbibliothek Winterthur, für die findige Beschaffung besonderer Literatur, sowie den Bibliotheken UB Mainz, St. B. Augsburg und ZB Zürich und Luzern für die prompte Besorgung von Photokopien aus nicht ausleihbaren Großlexica.

In der Hauptsache jedoch habe ich vor allem Herrn Dr. H. Haffter in Winterthur, em. Prof. an der Universität Zürich, für entscheidende Anregung und begleitende Beratung meinen verbindlichsten Dank zu bekunden. 


\section{Summary}

In his first account (Quarterly "Linnaea" I, 1826) of the "Rurik" research expedition which the former Chancellor of the Russian Empire, Romanzow, under Czar Alexander I dispatched with the mission of exploring the Bering Strait, the expedition's natural scientist, Chamisso, uses the phrase "Favente Salvinavia" ("with the blessing of Salvinavia") in his description of their departure from Copenhagen, a not uncommon phrase, although with a striking name. After having taken on the task of researching if Salvinavia was a Roman goddess, I found out that no such goddess exists.

However, quite different investigation led me to three stone altars discovered in the old Tiber wharf in the early eighteenth century, showing inscriptions with the name of the goddess (Cybele) Navisalvia (see picture). There is no other record of this name.

Since then it has been my opinion that Chamisso must have known this Latin word occurring for the first time not long before and created a new name by interchanging the component parts of the original.

The facts that Chamisso mastered the classical languages, was knowledgeable in ancient literature and, like other romanticists of his time, was greatly interested in the structure of the languages, may serve to support my conclusion. Further can be seen a topical relationship between Chamisso's Salvinavia passage and the event, often treated in literature, alluded to in the Navisalvia Inscription (CIL VI, 492 = Dessau 4096) together with the relief belonging to it, i.e. the seafaring of goddess Cybele from Asia Minor to Rome, the famous religious act inaugurating the great victory over Hannibal. Chamisso's literary source is doubtless Ovidius, Fasti IV, 247-348, where the event is described in detail, together with IV, 157-162, where Venus is honoured as Verticordia (= quae corda vertit), a word-formation serving as exact model for Chamisso's neologism Salvinavia (= quae naves salvat).

Finally the process of how the newly discovered word Navisalvia made its way into classical scholarship as well as the history of the then revived knowledge of its historic background tend to confirm my thesis.

Dr. Carl Arbenz

Rychenbergstraße 113

CH-8400 Winterthur 